\title{
REVIEW
}

\section{Intelligent Operating Theater Using Intraoperative Open-MRI}

\author{
Hiroshi Iseki ${ }^{1,2 *}$, Yoshihiro Muragaki ${ }^{1}$, Ryoichi Nakamura ${ }^{1}$, Norihiko Ozawa ${ }^{1}$, \\ Hiroki TANIGUCHI ${ }^{1}$, Tomokatsu HORI ${ }^{2}$, and Kintomo TAKAKURA ${ }^{1}$ \\ ${ }^{1}$ Faculty of Advanced Technosurgery, Division of Advanced Biomedical Engineering \& Science, \\ Graduate School of Medicine, Institute of Advanced Biomedical Engineering \& Science, \\ ${ }^{2}$ Department of Neurosurgery, Tokyo Women's Medical University \\ 8-1 Kawada-cho, Shinjuku-ku, Tokyo 162-8666, Japan
}

(Received September 1, 2005; Accepted September 29, 2005)

\begin{abstract}
Malignant brain tumors vary among patients and are characterized by their irregular shapes and infiltration. Localization of functional areas in the brain also differs among patients, and excess removal of tumor near eloquent areas may increase the risk of damage of function, such as motor paresis and speech disturbance. Recent progress in magnetic resonance (MR) imaging technology has enabled acquisition of intraoperative images and totally changed the neurosurgery of malignant brain tumors. Before, surgeons could merely speculate about the results of surgical manipulation and have no certainty about procedure outcomes until postoperative examination. Because intraoperative MR images allow visualization of the size of residual tumor(s) and the positional relationship between the tumor(s) and eloquent areas, surgeons are now able to achieve safe and reliable surgery. As an example, positional error on preoperative MR images caused by shifting of the brain (brain shift), a long-standing annoyance for surgeons, has been resolved using intraoperative MR images for surgical navigation, allowing precise resection. Two types of open-MR imaging scanner, a 0.2- or 0.3-tesla hamburger-type scanner with a horizontal gap and a 0.12 - or 0.5 -tesla double doughnut-type scanner with a vertical gap, are now available in the operating theater, and 1.5-tesla bore-type scanners are available. A 3.0-tesla bore-type scanner is planned. Intraoperative MR imaging includes diffusion-tensor and diffusionweighted imaging, which allows visualization of nerve fibers in the white matter, especially the pyramidal tract. Such images are valuable aids in the precise resection of residual lesions of malignant brain tumors near eloquent areas without injuring motor function.
\end{abstract}

\section{Keywords: intraoperative MRI, brain shift, update navigation, diffusion-weighted image, pyramidal tract}

\section{Introduction}

Malignant brain tumors vary among patients and are characterized by their irregular shapes and patterns of infiltration. Localization of functional areas in the brain also differs among patients, and excess removal of tumor near eloquent areas may increase the risk of damage to function, such as motor paresis and speech disturbance. Quality of therapy (QOT) enhances quality of life (QOL), and in neurosurgery, QOT, and thus QOL, will be improved by the most extensive preservation of postoperative brain function and maximization of

\footnotetext{
*Corresponding author, Phone: +81-3-3353-8111 ex 39989,
} Fax: +81-3-3341-0613, E-mail: hiseki@abmes.twmu.ac.jp resection rate of tumor possible. Establishing an intelligent operating theater improves QOT to assure secure and safe surgery, thus improving QOL for patients by limiting risks of motor dysfunction.

Sharing among surgical staff of surgical information on intraoperative magnetic resonance (MR) images enables rapid communication and increases the surgeon's knowledge of the condition of focus and its surrounding area. In the case of a malignant brain tumor, a resection rate of 90 to $95 \%$ can be achieved safely and certainly when the surgeon can check the residual tumor on intraoperative MR images. 


\section{Configuration of the operating theater practice}

In our operating theater ( $5.8 \mathrm{~m}$ by $4.2 \mathrm{~m})$, a vertical magnetic field ( 0.3 tesla), permanent magnet, open-MRI scanner with $43 \mathrm{~cm}$ gap (AIRIS $\mathrm{II}^{\mathrm{TM}}$, Hitachi Medical Co., Chiba, Japan) was installed. The theater is densely packed and relatively narrow compared to ordinary operating theaters, and the many surgical devices are managed to conform to the narrow space for effective and efficient use so that the surgeon and surgical staff can perform surgery with less movement. Because the area of magnetic leakage (5-gauss line) in which a pacemaker is affected is limited, the surgical staff can use conventional surgical devices and instruments advantageously in this small place without problems with the magnetic field. In addition, because the MRI scanner does not require a cooling system, its cost of operation is extremely low. ${ }^{1-3} \mathrm{~A}$ surgical coil system integrated with a head fixation frame improves both imaging performance and operation during surgery and provides surgical staff with high quality intraoperative MR images. With the surgical coil system, the surgeon can fix the patient's head at the most desirable position to acquire optimum diffusion-weighted (DW) images with less image distortion irrespective of the patient's orientation. Further, if needed, use of a stereotactic surgical device enables the surgeon to rapidly diagnose a biopsy sample (Fig. 1). ${ }^{4}$

On 5 displays in the operating theater, images of $\mathrm{MR}$, microscope or endoscope, and/or navigation, awake-surgery support, or physiological function are monitored via switching among 16 chargecoupled device cameras within the theater. The surgical staff can share all aspects of the surgical information via the 5 monitoring devices. Preoperatively, consolidation of the 3-dimensional medical images enables the surgical staff to visualize the structure of the targeted brain tumor and its surrounding area, especially the location of nerve fibers such as the pyramidal tract (motor fiber bundle). Intraoperatively, a surgical navigation system shows the surgical staff positional information for precision manipulation within less than $1 \mathrm{~mm}$. When intraoperative brain shift (brain deformation or movement caused by surgical manipulation) renders preoperative images imprecise, intraoperative MR imaging renews the navigation information and supports precision and safety of surgery. Intraoperative images show surgical staff precise and clear information about residual tumor and provide them security to assist in decision making for the next step.

\section{Surgical operating system}

Neurosurgery under open MRI can be compared with F1 racing. That is, rapid pit work (intraoperative MRI) allows rapid return of the F1 machine (the surgical team) to the course (surgery). The surgeon acquires an intraoperative MR image using the open-MRI scanner to confirm brain deformation caused by surgical procedures and tumor situation. Next, the surgical staff sends the image to a computer for intraoperative update navigation, and the surgical team continues the surgery aided by the updated navigation information. Surgery under open MRI is completed by repeating these procedures. The problem becomes the duration and labor of the interruption of surgery by the intraoperative open MRI. It is important to reduce the number and duration of pit stops (Fig. 2).

\section{Compensation for brain shift by update navigation}

The biggest issue during navigation surgery is the brain shift caused by surgical procedures (removal of tumor, suction of cerebrospinal fluid). ${ }^{5}$ In navigation using preoperative images, even in early surgery, the position of the target to be removed is no longer accurate on the navigation screen, and the gap between the real target and the target in the navigation image increases as surgery advances. The surgeon performs surgery taking the shift into consideration. It is extremely difficult to remove maximum tumor if the tumor exists near an eloquent area, but use of intraoperative images for navigation enables precise location of the target after brain shift appears on the navigation screen so that the surgeon can safely remove the tumor under navigation. ${ }^{6}$

The average error for update navigation is 0.93 $\mathrm{mm}$ with the availability of a marker system that can be used for both $\mathrm{T}_{1}$ - and $\mathrm{T}_{2}$-weighted imaging (Fig. 3.). ${ }^{7}$ A pit-in system with which an operating table and machine table was easily separable was also developed. Therefore, the amount of time needed for one set of intraoperative MRI (to interrupt the surgery, to move the operating table, to acquire an image, and to re-start the surgery) was cut markedly. It takes 5 min each for the preparation for imaging, for one cool of imaging (either for $\mathrm{T}_{1^{-}}$or $\mathrm{T}_{2}$-weighted imaging), and for restarting of surgery (total time of $15 \mathrm{~min}$ compared to 30 min before the introduction of the pit-in system). 


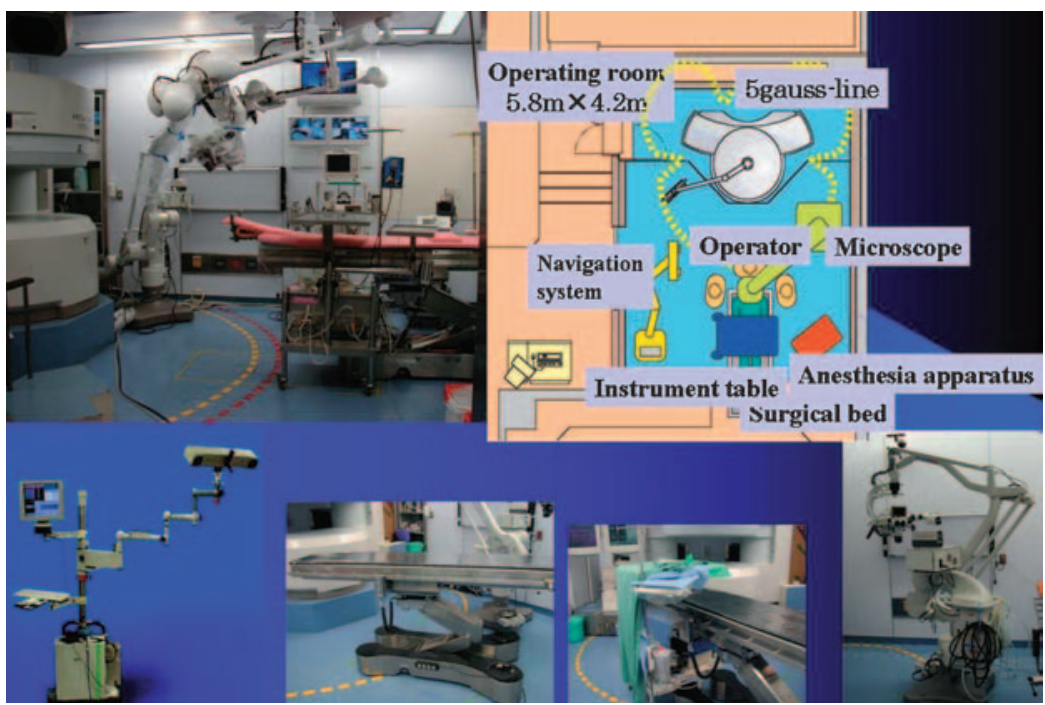

Fig. 1. Outline and surgical modalities and devices of our intelligent operating theater.

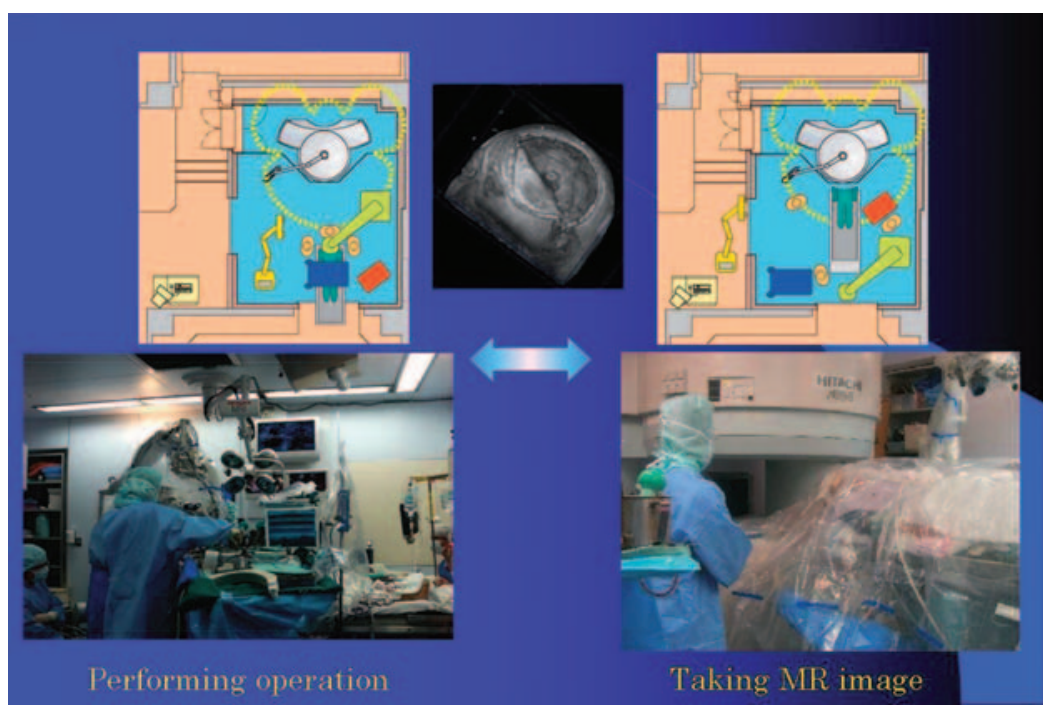

Fig. 2. Pit-in procedure and system (left panel: surgical procedure; right panel: acquisition of intraoperative magnetic resonance image). Note: Yellow dotted line indicates the 5-gauss line.

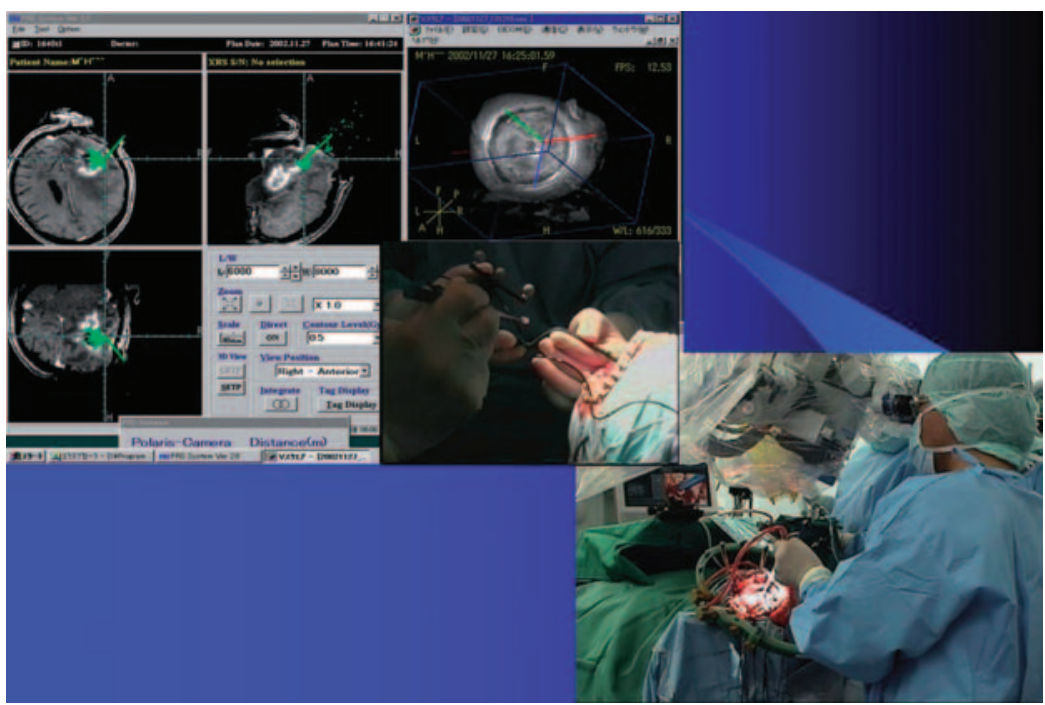

Fig. 3. Update navigation based on intraoperative multi-slice magnetic resonance images. 


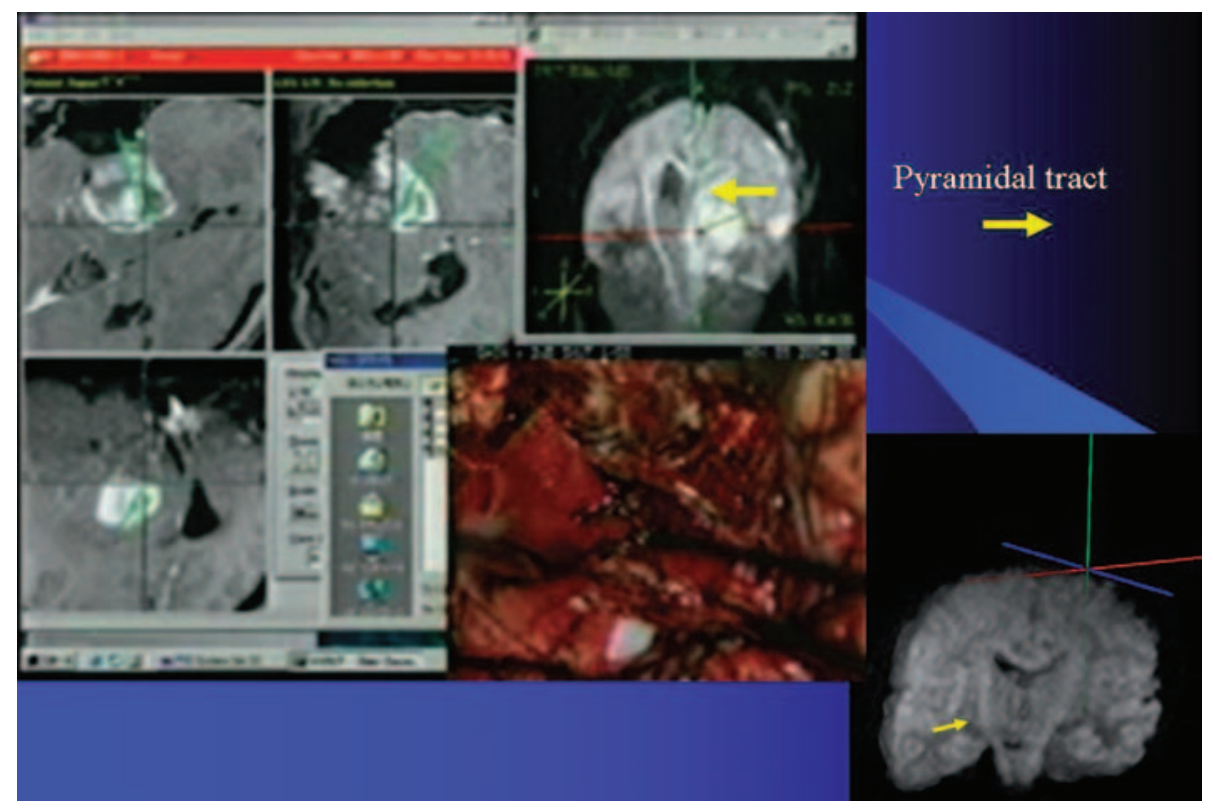

Fig. 4. Update navigation and intraoperative diffusion-weighted image navigation for the resection of a malignant brain tumor adjacent to pyramidal tract.

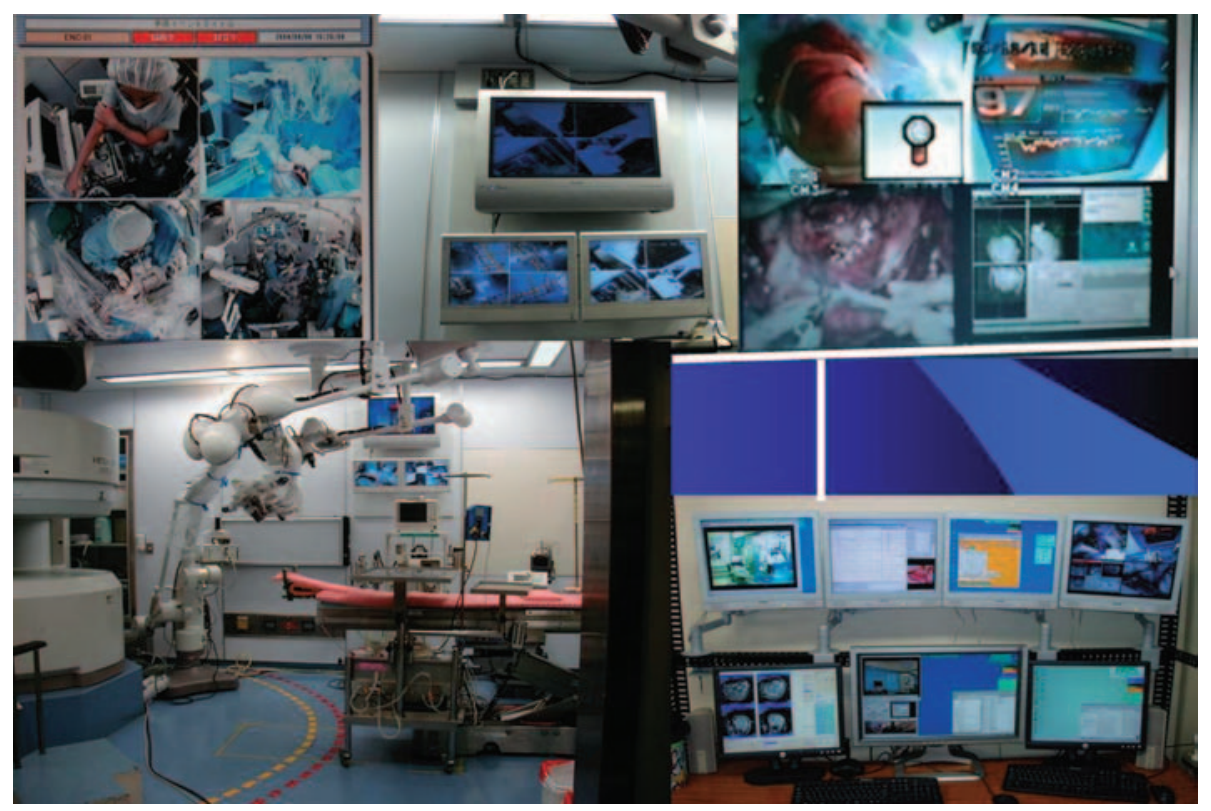

Fig. 5. Integrated surgical information on multiple displays during surgery.

\section{Surgery near the pyramidal tract aided by diffusion-weighted image navigation}

DW imaging enables successful visualization of the pyramidal tract, and compared to high magnetic field MRI, DW imaging has low image distortion and is therefore superior to ordinary MRI in depicting the positional information of the pyramidal tract. A report at the Computer Assisted Radiology and Surgery International Congress and
Exhibition in Berlin in 2005 indicated that damage from image distortion could be alleviated by using a wide margin around the pyramidal tract on the tractogram acquired by MRI with high magnetic field strength for navigation. ${ }^{8}$ Visualization of the pyramidal tract on a 3-dimensional DW image with low distortion and use of the image to update navigation to compensate for the brain shift enabled the surgeon to perform radical resection of malignant tumor near the pyramidal tract (Fig. 4). ${ }^{9}$ 


\section{Preservation of brain function under awake surgery}

For surgery of malignant tumor located near functional areas (especially the speech and motor areas), preservation of function is important. Because a malignant tumor has an irregular shape and infiltration pattern and arises from the brain parenchyma, it is difficult to distinguish the lesion from surrounding normal brain tissue. To preserve significant brain function for the patient, the surgeon must consider that functional brain area varies among patients and may be compressed by the migration of the tumor. Further, as tumor is removed more radically, the possibility of squeals could increase. For awake surgery, it is essential in intraoperative physiological monitoring to log tasks and precisely record and analyze the patient's responses to the tasks. Although the surgeon, the anesthesiologist, and other supporting personnel work independently, they must cooperate and work as a team. Sharing of all information through the use of an intraoperative examination monitor for awake surgery system that simultaneously displays the results of physiological monitoring, images of the surgical field, monitoring of the anesthetic condition, and navigation data on 5 monitor screens facilitates the close cooperation of the entire surgical staff (Fig. 5).

\section{Effects of development of various operating theaters}

Our ability to fuse diagnosis and treatment in our operating theater has increased the resection rate of malignant brain tumor up to $94 \%$ on average; the total resection rate has increased to $43 \%$ compared to the $8 \%$ indicated in the Report of Brain Tumor Registry of Japan (1969-1993) (RBTRJ). ${ }^{10}$ According to the RBTRJ, resection rate is the main determinant of the outcome of surgery in this disease. A 5-year survival rate is 14.8 to $10.8 \%$ when the resection rate is less than $75 \%, 22.5 \%$ when the rate is over $95 \%$, and $40.9 \%$ when the tumor is completely removed. However, if one puts much value on the increment of resection rate, severe functional impairment may result from damage to eloquent areas, such as those controlling motor and speech function. For this reason, it is very difficult to achieve a high resection rate without the help of awake surgery and/or intraoperative MR imaging and navigation. In our facility, 323 neurosurgeries were performed safely from March 2000 to August 2005 (Table 1), and the 4-year survival rates for Grade II glioma and for
Table 1. Neurosurgical cases in our intelligent operating theater between March 2000 and August 2005 (323 cases)

\begin{tabular}{lr}
\hline \multicolumn{1}{c}{ Tumor } & number of cases \\
\hline Glioma in eloquent area & 222 \\
Parasellar tumor (transnasal approach) & 40 \\
in pituitary tumor & 32 \\
in craniopharyngioma & 8 \\
Hydrocephalus & 3 \\
Arteriovenous malformation & 7 \\
Cavernous angiomas & 20 \\
Meningioma & 5 \\
Others & 26 \\
Technique used & \\
Navigation & 242 \\
Navigation with DWI* & 6 \\
Awake surgery & 47 \\
5-ALA enhancement** & 121 \\
\hline
\end{tabular}

*DWI: Diffusion-weighted image

**5-ALA: 5-aminolivulinic acid

Grade III glioma were $100 \%$ and $80 \%$, respectively, improving dramatically from the rates of $70 \%$ and $27 \%$ reported in the RBTRJ, 1969-1993 (Table 2). According to a study on financial impact by Schulder and associates, in comparison with other image-aided modalities, the introduction of an intraoperative MRI ( 0.15 tesla) considerably reduced hospital stay and complications of patients with intracranial tumor. ${ }^{11}$ Between 1997 and 1998, Hall's group clinically applied a high magnetic field strength (1.5 tesla), short bore-type, helium-cooled magnet (Philips Gyroscan ACS-NT ${ }^{\mathrm{TM}}$, Philips Medical Systems, Best, The Netherlands) in 101 neurosurgeries and found its intraoperative use was a safe and effective technique to support neurosurgery. In 24 of 30 patients ( $80 \%$ ), total resection was achieved using a radiological technique because it allowed intraoperative verification of residual tumor. This preliminary result indicates that intraoperative MRI has a significant influence on the intraoperative decision-making of the surgeon. ${ }^{12}$ Wirtz and associates analyzed intraoperative images and surgical outcome in 97 patients who underwent upper tentorial glioma treatments among 242 intraoperative MRI-guided surgeries. The rate of patients demonstrated to have residual tumor was significantly reduced by intraoperative MRI from $62 \%$ after the first resection to $33 \%$ after surgery. The authors reported that the updating of navigation data with intraoperative MR images alleviated the problems caused by the brain shift, assuring safe and reliable surgery and contributing significantly to the maximization of 
Table 2. Surgical outcome of malignant brain tumor (glioma) in our intelligent operating theater

\begin{tabular}{|c|c|}
\hline \multicolumn{2}{|l|}{ Removal rate (\%) } \\
\hline Average removal rate & 94 \\
\hline Total removal rate & 41 \\
\hline \multicolumn{2}{|l|}{ Comparison of 4-year survival rate (\%) } \\
\hline Astrocytoma (grade II) & $100\left(69.6\right.$ in RBTRJ $\left.^{*}\right)$ \\
\hline Anaplastic astrocytoma (grade III) & 80 (27.0 in RBTRJ) \\
\hline
\end{tabular}

*RBTRJ: Report of Brain Tumor Registry of Japan $(1969-1993)^{10}$

resection rate of tumor and prolongation of survival rate. ${ }^{13}$

\section{Intraoperative MRI operating theater in the world}

In 1995, a 0.5-tesla superconducting MRI system (SIGNA SP ${ }^{\mathrm{TM}}$, General Electric Medical Systems, Boston, MA, USA) was installed for neurosurgery at Brigham \& Women's Hospital, Harvard Medical School, for the first time ever. Surgery requires that the surgeon get into the $56-\mathrm{cm}$ gap of the gantry, a narrow space allowing only one surgeon to work in the case of surgery of the parietal region or two for surgery of the temporal region. The concept of this scanner emphasizes the need of real-time acquisition of intraoperative images by placing the patient continuously in the gantry although operationality is sacrificed somewhat. The magnetostatic field strength is 0.5 tesla, but the magnetic field strength of each doughnut is 1.5 tesla and is within the 20gauss line at the lowest inside the operating theater. The 5-gauss line lies outside the operating theater. Because all the essential surgical devices are to be used within the 20-gauss line, it is necessary to make all these devices MR compatible. However, such devices are very expensive, and their quality is not always satisfactory for surgery, especially when the patient's bed is used exclusively in the gantry and cannot then serve as operating table as well. Further, the table can be moved only vertically or horizontally. At present, Brigham \& Women's Hospital is constructing a novel operating theater in which a 3-tesla MRI scanner (General Electric Medical Systems) and a positron emission tomography/flat panel digital subtraction angiography scanner are to be installed as next generation modalities. ${ }^{14}$

In Japan, a double doughnut-type open-MRI scanner was introduced in 2000 at Shiga University of Medical Science and has been used mainly for treating the liver.
In 1997, at Erlangen-Nuremberg University, a twin operating theater was constructed in which a 0.2-tesla, hamburger-type, normal conduction magnet, open-MRI scanner (Magnetom Open ${ }^{\mathrm{TM}}$, Siemens AG, Erlangen, Germany) was installed, and it was used until 2003. Because this operating theater was designed under special consideration of compatibility with a conventional surgical system, the MRI scanner had a narrow 5-gauss line so that conventional surgical devices and instruments could be used..$^{15}$ In 2003, a 1.5-tesla, bore-type MRI scanner (Magnetom Sonata Maestro Class ${ }^{\mathrm{TM}}$, Siemens AG, Erlangen, Germany) was installed as a successor system and is now in use clinically. ${ }^{16}$ Compared to an open-MRI scanner, the bore-type has limited working space, and consequently, surgical manipulation should be done outside the gantry (open space). A head fixation frame is attached to the inside of a housing-type head coil and can be swung or tilted. The table for surgery can be rotated to move into the cylinder-type MR scanner for image acquisition. After its return to the surgical position, the table's height is adjusted according to the surgeon's body size. An anesthetic device is fixed on a pole and can be rotated around the pole. Conventional surgical devices and instruments can be used advantageously outside the 5gauss line, and only those carried over in the gantry must be MR compatible. However, special caution is needed to avoid carryover of conventional surgical devices and instruments within the 5-gauss line.

Since 1997, at the University of Minnesota, a high magnetic field strength (1.5 tesla), short boretype MRI scanner (Gyroscan ACS-NT ${ }^{\mathrm{TM}}$, Philips Medical Systems) has been in use. ${ }^{17}$ This scanner has a helium cooling system.

At the University of Calgary, in collaboration with Innovative Magnetic Resonance Imaging System, Inc. (Winnipeg, Manitoba, Canada), a high magnetic field strength (1.5 tesla), bore-type, original mobile-type MRI scanner weighing 6 metric tons was installed under the concept that the gantry rather than the patient should be moved. ${ }^{18}$

PoleStar $^{\mathrm{TM}} \mathrm{N}-10$ (Odin Medical Technologies, Yokneam, Israel) is a mobile-type, 0.12-tesla, open-MRI scanner weighing $460 \mathrm{~kg}$ with a $25-\mathrm{cm}$ vertical gap. This system was designed exclusively for neurosurgery to obtain brain images. Its biggest selling point was its easy setting in existing operating theaters. However, this system required simplified radiofrequency shields and lost its appeal..$^{19,20}$

Compared to low magnetic field MRI scanners, high magnetic field scanners have a wide margin for the 5-gauss line and therefore involve too many difficulties in choosing surgical procedures, devices, 
and instruments. Cost performance is also poor. Under such circumstances, the kind of surgery indicated for the high magnetic field scanner is uncertain. In contrast, for low magnetic field MRI scanners, the challenge is the extent to which the quality of diffusion-tensor (DT) and DW images with low distortion can be improved. Nevertheless, the selection criteria must balance the requirements of surgical staff and the performance of each MRI scanner.

\section{Discussion}

There is no question about the usefulness of intraoperative images for surgery. At the moment, ultrasonography, computed tomography (CT), and magnetic resonance imaging are used for intraoperative diagnostic imaging. Diagnostic ultrasound is cost effective and easy to use and provides real-time images. However, image quality is limited to confirming residual tumors because of the high echo around the peripheral region of the cavity after tumor resection. To compensate for the poor image quality, integration of a navigation system using preoperative CT/MRI images with echograms has been attempted. However, this does not always overcome the problem of shifting of the brain. With respect to CT, the number of plane images obtainable in a single scan increases and the distortion of CT images is far less than that of MR image. Ignoring exposure to radiation, CT can provide any kind of slice image, and image quality is satisfactory except for soft tissue. The problem is how much imaging should be allowed to remove as much tumor as possible near functional areas when more imaging results in more exposure to radiation for the patient. MRI exhibits good imaging power and high image quality of soft tissue without radiation exposure. Image distortion for $\mathrm{T}_{1^{-}}$and $\mathrm{T}_{2}$-weighted images varies by magnetic strength (low, intermediate, and high), but remains within practical ranges. When image quality and production of functional MR images are prioritized, a high magnetic field MRI scanner is superior to one with a lower magnetic field because of the high signal-to-noise $(\mathrm{S} / \mathrm{N})$ ratio. The system needed depends on the kind of images required. For ordinary images used for surgery, low-magneticfield and higher-magnetic-field scanners produce images of comparable quality. The high magnetic field (1.5 tesla) MRI scanner is excellent for taking DT images as a result of the high $\mathrm{S} / \mathrm{N}$ ratio, although the image has large distortion. The surgeon avoids complications, such as motor paralysis, by setting a wider margin around the tractogram while considering the distortion error. ${ }^{1}$ The surgeon checked that error of location of the pyramidal tract on DW image with low distortion was small by means of intraoperative electrical stimulation to the white matter. Unlike tractography, DTI has advantages in that it requires no particular image processing so that the surgeon can restart surgery immediately after the image data is transferred to a navigation computer by the local area network. At any rate, visualization of the pyramidal tract provides physiological and anatomical information that serves as a valuable intraoperative resource for the surgeon. Regarding DW images, the distortion of low or intermediate magnetic field strength is superior to that of high magnetic field and vice versa for image quality. It is merely the surgeon's choice whether a high quality image of the fiber bundle is to be used at the sacrifice of distortion of location or an image with low distortion is to be used though image quality is somewhat poor. Nimsky and associates accommodated the width of the tractogram shown on navigation using high magnetic field (1.5 tesla) tractography. ${ }^{9}$ Using a 0.3 -tesla open-MRI scanner to obtain a DW image for navigation, the authors confirmed small distortion of the pyramidal tract location using electrophysiological monitoring of motor evoked potential. ${ }^{8}$ Gasser's group identified functional areas by performing intraoperative functional MRI (1.5 tesla) by median nerve stimulation rather than identifying function using awake surgery. ${ }^{21}$ Some investigators identified functional areas using intraoperative functional MR images acquired by low magnetic field ( 0.12 tesla), mobiletype open-MRI scanner (PoleStar $^{\mathrm{TM}} \mathrm{N}-10$, Odin Medical Technologies). ${ }^{22}$ The authors confirmed the functional areas with pinpoint precision by direct electrical stimulation of the cortex during awake surgery. Therefore, we need no functional MRI for the speech area with respect to accuracy. Regarding the motor area, we are trying to use functional MRI, too.

\section{References}

1. Iseki H, Muragaki Y, Taira T, et al. New possibilities for stereotaxis. Information-guided stereotaxis. Stereotact Funct Neurosurg 2001; 76:159-167.

2. Iseki $\mathrm{H}$, Muragaki $\mathrm{Y}$, Takakura $\mathrm{K}$, et al. MRI clinical techniques. Know-how in treatment: points in intraoperative MRI. Innervision JIYUKUKAN 2002; 8-11.

3. Iseki $\mathrm{H}$, Muragaki $\mathrm{Y}$, Naemura K, Hayashi $\mathrm{M}$, Hori T, Takakura K. Clinical application of augmented reality in neurosurgical field, In: Proceedings of Computer Graphics International. 
Tokyo, Japan, 2003; 44-49.

4. Taniguchi H, Iseki H, Taira $\mathrm{T}$, et al. Development of Hitchcock stereotactic frame for intraoperative open MRI, In: Lemke HU, Doi K, Farman AG, Inamura K, Reiber JHC, Vannier MW, eds. Proceedings of the 16th International Congress and Exhibition of Computer Assisted Radiology and Surgery. Paris, France, 2002; 144-149.

5. Nimsky C, Ganslandt O, Cerny S, Hastreiter P, Greiner G, Fahlbusch R. Quantification of, visualization of, and compensation for brain shift using intraoperative magnetic resonance imaging. Neurosurgery 2000; 47:1070-1079; discussion 1079-1080.

6. Wirtz CR, Tronnier VM, Bonsanto MM, et al. Image-guided neurosurgery with intraoperative MRI: update of frameless stereotaxy and radicality control. Stereotact Funct Neurosurg 1997; 68: 39-43.

7. Sugiura M, Muragaki Y, Nakamura R, Hori T, Iseki H. Accuracy evaluation of an update-navigation system for the resection surgery of brain tumor using intraoperative magnetic resonance imaging. Journal of Japan Society of Computer Aided Surgery 2005; 7:43-49. (Japanese)

8. Ozawa N, Muragaki Y, Shirakawa H, Suzukawa H, Nakamura R, Iseki H. Navigation system based on intraoperative diffusion weighted imaging using open MRI, In: Lemke HU, Inamura K, Doi K, Vannier MW, Farman AG, eds. Proceedings of the 19th International Congress and Exhibition of Computer Assisted Radiology and Surgery. Berlin, Germany, 2005; 810-814.

9. Nimsky C, Ganslandt $O$, Hastreiter $P$, et al. Preoperative and intraoperative diffusion tensor imaging-based fiber tracking in glioma surgery. Neurosurgery 2005; 56:130-137; discussion 138.

10. The Committee of Brain Tumor Registry of Japan. Report of brain tumor registry of Japan (19691993) 10th ed [editorial]. Neurol Med Chir 2000; 40 Supple:54.

11. Schulder M, Ankitkumar P. Financial impact of intraoperative MRI. Final program of 14th Meeting of the World Society for Stereotactic and Functional Neurosurgery, Rome, Italy, 2005.

12. Martin AJ, Hall WA, Liu H, et al. Brain tumor resection: intraoperative monitoring with highfield-strength MR imaging-initial results. Radiolo- gy $2000 ; 215: 221-228$.

13. Wirtz CR, Knauth M, Staubert A, et al. Clinical evaluation and follow-up results for intraoperative magnetic resonance imaging in neurosurgery. Neurosurgery 2000; 46:1112-1120; discussion 1120-1122.

14. Black PM, Moriarty T, Alexander E 3rd, et al. Development and implementation of intraoperative magnetic resonance imaging and its neurosurgical applications. Neurosurgery 1997; 41:831-842; discussion 842-845.

15. Steinmeier R, Fahlbusch R, Ganslandt O, et al. Intraoperative magnetic resonance imaging with the Magnetom open scanner: concepts, neurosurgical indications, and procedures: a preliminary report. Neurosurgery 1998; 43:739-748.

16. Nimsky C, Ganslandt O, Von Keller B, Romstock J, Fahlbusch R. Intraoperative high-field-strength MR imaging: implementation and experience in 200 patients. Radiol 2004; 233:67-78.

17. Hall WA, Liu H, Martin AJ, Pozza CH, Maxwell RE, Truwit CL. Safety, efficacy, and functionality of high-field strength interventional magnetic resonance imaging for neurosurgery. Neurosurgery 2000; 46:632-641; discussion 641-642.

18. Kaibara T, Saunders JK, Sutherland GR. Advances in mobile intraoperative magnetic resonance imaging. Neurosurgery 2000; 47:131-137; discussion 137-138.

19. Hadani M, Spiegelman R, Feldman Z, Berkenstadt $H$, Ram Z. Novel, compact, intraoperative magnetic resonance imaging-guided system for conventional neurosurgical operating rooms. Neurosurgery 2001 ; 48:799-807; discussion 807-809.

20. Levivier M, Wikler D, De Witte O, Van de Steene A, Baleriaux D, Brotchi J. PoleStar N-10 low-field compact intraoperative magnetic resonance imaging system with mobile radiofrequency shielding. Neurosurgery 2003; 53:1001-1006; discussion 1007.

21. Gasser T, Ganslandt O, Sandalcioglu E, Stolke D, Fahlbusch R, Nimsky C. Intraoperative functional MRI: implementation and preliminary experience. Neuroimage 2005; 26:685-693.

22. Schulder M, Azmi H, Biswal B. Functional magnetic resonance imaging in a low-field intraoperative scanner. Stereotact Funct Neurosurg 2003; 80:125-131. 